\title{
Neuroprotective effects of syringic acid against OGD/R-induced injury in cultured hippocampal neuronal cells
}

\author{
YIDONG CAO $^{1 *}$, LIANG ZHANG ${ }^{1 *}$, SHUKAI SUN $^{1}$, ZHENHENG YI $^{2}$, XUE JIANG ${ }^{1}$ and DONG JIA ${ }^{1}$ \\ ${ }^{1}$ Department of Neurosurgery, Tangdu Hospital, The Fourth Military Medical University, Xi'an, Shaanxi 710053; \\ ${ }^{2}$ Department of Neurosurgery, The Second People's Hospital of Xinxiang City, Xinxiang, Henan 453002, P.R. China
}

Received October 6, 2015; Accepted May 13, 2016

DOI: 10.3892/ijmm.2016.2623

\begin{abstract}
Cerebral ischemic injury and treatment are important topics in neurological science. In the present study, an in vitro model of cerebral ischemia was established by subjecting primary cultures of hippocampal neuronal cells to oxygen-glucose deprivation followed by reperfusion (OGD/R), in order to evaluate the possible neuroprotective role of syringic acid (SA). The results of 3-(4,5-dimethylthiazol-2-yl)2,5-diphenyltetrazolium bromide (MTT) and lactate dehydrogenase (LDH) assays showed that pre-treatment with SA $(0.1,1,10$, and $20 \mu \mathrm{M})$ attenuated $\mathrm{OGD} / \mathrm{R}$-induced neuronal injury in a dose-dependent manner, with evidence of increased cell viability and decreased LDH leakage. In addition, oxidative stress markers were evaluated using commercial kits, and the results demonstrated that $\mathrm{OGD} / \mathrm{R}$ exposure induced distinct oxidative stress, accompanied by elevated levels of intracellular reactive oxygen species (ROS) and malondialdehyde (MDA) production, and reduced activity of the antioxidant enzyme superoxide dismutase (SOD), which were dose-dependently restored by pre-treatment with SA. In addition, the concentration of intracellular free calcium $\left[\mathrm{Ca}^{2+}\right]_{\mathrm{i}}$ and mitochondrial membrane potential (MMP or $\Delta \psi_{\mathrm{m}}$ ) were determined in order to evaluate the degree of neuronal damage by performing flow cytometric analysis and observing the cells under a fluorescence microscope, respectively. We demonstrated that pre-treatment with SA inhibited elevations in $\left[\mathrm{Ca}^{2+}\right]_{i}$, whereas it increased the MMP dose-dependently following exposure to OGD/R. Western blot analysis revealed that $\mathrm{OGD} / \mathrm{R}$ promoted cell apoptosis with concomitant increases in Bax and caspase- 3 expression, and reduced Bcl-2 expression, which was reversed by pre-treatment with SA in a dose-dependent manner. Moreover, these effects were mediated through the JNK and p38 pathways, as
\end{abstract}

Correspondence to: Dr Dong Jia, Department of Neurosurgery, Tangdu Hospital, The Fourth Military Medical University, 1 Xinsi Road, Baqiao, Xi'an, Shaanxi 710053, P.R. China

E-mail: jiadong_neuro@126.com

*Contributed equally

Key words: syringic acid, oxygen-glucose deprivation and reperfusion, cell injury, hippocampal neurons pre-treatment with SA inhibited the OGD/R-induced increase in phosphorylated (p-)JNK and p-p38 expression. Taken together, these results suggested that SA exerted strong neuroprotective effects in hippocampal neuronal cells, which may be attributed to the attenuation of OGD/R-induced cell injury through the JNK and p38 signaling pathways.

\section{Introduction}

Ischemic stroke is a major cause of death and is associated with high rates of morbidity and disability in adults worldwide (1). As an acute cerebrovascular disease, it is associated with cerebral ischemia and brain tissue damage due to significant deprivation of oxygen and glucose caused by a reduction in or complete blockade of arterial blood supplies to the brain (2). Transient cerebral ischemia induced by the deprivation of blood to the brain may cause delayed neuronal death in some specific vulnerable regions such as the hippocampus (3). Early restoration of cerebral blood flow is crucial for sustaining neuronal viability. Nevertheless, reperfusion is believed to promote delayed secondary brain injury as the freshly arriving oxygen will serve as a substrate for the production of excessive reactive oxygen species (ROS). Thus, antioxidant defenses including free radical scavengers and antioxidant enzymes are considered a promising approach to reduce the extent of damage caused by ischemia/reperfusion injury.

Cumulative evidence suggests that oxidative stress is a fundamental mechanism of cerebral ischemia/reperfusion injury (2). Oxidative stress and mitochondrial dysfunction are frequently implicated in the pathology of secondary neuronal damage following cerebral ischemia/reperfusion through the generation of ROS $(4,5)$. Mitochondria are abundant in cerebral tissues, and are a major source of cerebral intracellular ROS, and the imbalance between the generation and degradation of ROS leads to oxidative stress (6). Moreover, the overall process of ischemia/reperfusion injury is extremely complex. Cell injury induced by ischemia/reperfusion is a multifactor, multimechanism, malignant cascade reaction, which includes many events, such as the increased release of excitatory amino acids, calcium overload, mitochondrial membrane depolarization, free radical production, apoptosis gene activation and so forth. These factors reinforce each other and are interrelated, forming a vicious cycle, which eventually results in cell apoptosis or necrosis (7). For example, the enhancement of concentrations 
of intracellular free calcium $\left[\mathrm{Ca}^{2+}\right]_{\mathrm{i}}$ and reduction in mitochondrial membrane potential (MMP or $\Delta \psi_{\mathrm{m}}$ ) has been proven to contribute to the secondary mitochondrial dysfunction induced by cerebral ischemia/reperfusion injury in neonatal rat primary cultured hippocampal neurons (7). Thus, inhibition of oxidative stress and mitochondrial dysfunction is beneficial in the treatment of cerebral ischemia/reperfusion injury.

Polyphenols are found in many plants and possess immunomodulatory and anti-inflammatory effects, as well as antioxidant effects as they are capable of removing ROS formed by lipid peroxidation, cellular damage and oxidative stress (8). In particular, syringic acid (SA; 4-hydroxy-3,5-dimethoxybenzoic acid), a polyphenolic derivative of benzoic acid (9), has been shown to exert antioxidant, chemoprotective and antimicrobial effects $(9,10,11)$. Recent studies in a rat model of ischemia/reperfusion suggest that SA reduces oxidative stress $(9,12)$. However, the regulatory role of SA in a model of hippocampal neurons subjected to oxygen-glucose deprivation followed by reperfusion (OGD/R)-induced cell injury and furthermore, the potential mechanisms involved, have not yet been explored, to the best of our knowledge.

In the present study, we principally focused on the protective effects of SA in OGD/R-treated primary hippocampal neuronal cells by evaluating cell viability, oxidative stress markers, $\left[\mathrm{Ca}^{2+}\right]_{i}$, MMP and cell apoptosis, and by elucidating the associated molecular mechanisms. Nimodipine is a calcium antagonist, derived from the 1,4-dihydropyridine ring with a preferential cerebrovascular action (13). This drug is light-sensitive and also lipophilic, which enables it to cross the blood-brain barrier and enter the brain (14). It has been reported that nimodipine exerts potent cerebrovascular effects in vitro (15). Thus, in the present study, nimodipine was used as a positive control drug.

\section{Materials and methods}

Animals, reagents and antibodies. Male Sprague-Dawley (SD) rats $(\mathrm{n}=20), 220-240 \mathrm{~g}$, were purchased from Vital River Laboratory Animal Technology Co., Ltd. (Beijing, China). All reagent-grade chemicals, SA (purity $>95 \%$ ), dimethyl sulfoxide (DMSO), and 3-(4,5-dimethylthiazol-2-yl)-2,5-diphenyltetrazolium bromide (MTT) were purchased from Sigma (St. Louis, MO, USA). The neurobasal medium containing B27 supplements, fetal bovine serum (FBS), horse serum and other culture products was purchased from Gibco (Rockville, MD, USA). Nimodipine injection $(5 \mathrm{mg} / \mathrm{ml})$ was purchased from Bayer AG (Leverkusen, Germany). The lactate dehydrogenase (LDH) activity assay kit and the commercial kits for the detection of superoxide dismutase (SOD) and ROS were purchased from Jiangcheng Bioengineering (Nanjing, China). The commercial kits for the detection of MMP and malondialdehyde (MDA) content were purchased from Beyotime (Nanjing, China). Fluo-3-acetoxymethyl ester (Fluo-3 AM) was purchased from Biotium, Inc. (Hayward, CA, USA). Antibodies against phospho-p38 (\#4631) and phospho-JNK (\#4668) antibodies were purchased from Cell Signaling Technology (Beverly, MA, USA); JNK (sc-572), p38 (sc-535), caspase-3 (sc-1225) and Bax (sc-493) were obtained from Santa Cruz Biotechnology (Santa Cruz, CA, USA); Bcl-2 (\#D038-3) was purchased from MBL (Nagoya, Japan) and $\beta$-actin (A1978) was obtained from Sigma.
Horseradish peroxidase-conjugated anti-rabbit (A0208) or anti-mouse (A0216) immunoglobulin G, and enhanced chemiluminescence (ECL) reagents were purchased from Beyotime (Shanghai, China). The Pierce BCA protein assay kit was purchased from Thermo Fisher Scientific (Rockford, IL, USA). Polyvinylidene fluoride (PVDF) membranes were purchased from Millipore Corp. (Billerica, MA, USA).

Cell isolation and cell culture. Primary hippocampal neuronal cells were prepared from the brains of neonatal SD rats, as previously described (16) with some modifications. Briefly, the rats were sacrificed by decapitation and the hippocampal tissues were dissected on ice and then dissociated in $0.25 \%$ trypsin-EDTA. The primary hippocampal neurons were maintained in neurobasal medium supplemented with GlutaMAX and B27 plus glucose $(4.5 \mathrm{~g} / \mathrm{l})$ for 7 days. Then, the cells were cultured in a medium containing 5\% horse serum and 5\% FBS supplemented with $15 \mathrm{mM}$ glucose for 14 days. All cells were cultured in an incubator with a humidified atmosphere of $5 \% \mathrm{CO}_{2}$ at $37^{\circ} \mathrm{C}$. All experimental procedures were performed in accordance with the regulations of the Ethics Committee of Jilin University for the Care and Use of Laboratory Animals and were approved by the Institutional Animal Care and Use Committee (IACUC) of Jilin University (Changchun, China).

$O G D / R$ procedure. We established an in vitro model of cerebral ischemia by subjecting cells to OGD. The neurons were rinsed twice and incubated in DMEM without glucose. The culture media were then introduced into a specialized, humidified chamber filled with $1 \% \mathrm{O}_{2} / 94 \% \mathrm{~N}_{2} / 5 \% \mathrm{CO}_{2}$ at $37^{\circ} \mathrm{C}$ for $3 \mathrm{~h}$ in order to establish conditions of OGD. Thereafter, the culture media were replaced with normal DMEM containing glucose under normoxic conditions for an additional $24 \mathrm{~h}$ as OGD/R. SA $(0.1,1,10$, and $20 \mu \mathrm{M})$ or nimodipine (final concentration $5 \mathrm{mg} / \mathrm{l}$ ) was applied to the cell cultures $24 \mathrm{~h}$ prior to OGD/R. The cultures in the blank control group were maintained in normal DMEM medium under normoxic conditions at $37^{\circ} \mathrm{C}$ without OGD/R exposure and SA pre-treatment. The cultures in the control group were subjected to OGD/R in the absence of SA pre-treatment.

Cell viability assay. Neuronal cell viability was measured using the MTT assay. Briefly, the cultured cells were seeded into 96-well plates (Corning Inc., Corning, NY, USA) at a density of $5 \times 10^{3}$ cells/well in DMEM with $10 \%$ FBS for $24 \mathrm{~h}$ at $37^{\circ} \mathrm{C}$ in $5 \% \mathrm{CO}_{2}$. After exposure to OGD/R, $20 \mu \mathrm{l}$ modified tetrazolium salt MTT $(5 \mathrm{mg} / \mathrm{ml})$ was added to each well and the samples were incubated at $37^{\circ} \mathrm{C}$ for $4 \mathrm{~h}$. The supernatant was then carefully removed and $100 \mu \mathrm{l}$ DMSO was added to lyse the cells. Once the dark-blue MTT crystals had dissolved, absorbance was measured at $490 \mathrm{~nm}$ using a Benchmark microplate reader (Bio-Rad, Hercules, CA, USA).

Cytotoxicity assay. Neuronal injury in the cells was also quantitatively assessed by measuring the activity of LDH released from damaged or dead cells. Previous findings have shown that the activity of LDH from either necrotic or apoptotic cells is proportional to the number of neurons damaged or destroyed (17). Briefly, the cells were seeded in 96-well plates (Corning Inc.) at a density of $5 \times 10^{3}$ cells/well and incubated for 
$24 \mathrm{~h}$. After exposure to OGD/R, a $50 \mu \mathrm{l}$ volume of medium was removed, and the amount of LDH leakage from the cells was determined using the LDH activity assay kit according to the manufacturer's instructions. The absorbance of the samples was read spectrophotometrically at $490 \mathrm{~nm}$. The results are expressed as the percentage of $\mathrm{LDH}$ release relative to the control cells.

Measurement of cellular SOD and MDA levels. After exposure to $\mathrm{OGD} / \mathrm{R}$, the cells were harvested and resuspended in $0.1 \mathrm{M}$ ice cold PBS. The cell suspensions were sonicated for $25 \mathrm{sec}$ on ice and centrifuged at $12,000 \mathrm{rpm}$ at $4^{\circ} \mathrm{C}$ for $10 \mathrm{~min}$, and the supernatants were collected. SOD levels were assayed by a modification of the xanthine/xanthine oxidase method (18). Cellular SOD levels were determined by spectrophotometry. The levels of MDA, a compound produced during lipid peroxidation, were measured using the commercial kit based on a reaction with thiobarbituric acid (19). The optical density at $532 \mathrm{~nm}$ was determined using a microplate reader (Spectra Max 190; Molecular Devices, Sunnyvale, CA, USA).

Measurement of ROS content. ROS formation was determined using the fluorescent probe 2',7'-dichlorfluorescein-diacetate (DCFH-DA). Cell-permeable non-fluorescent DCFH-DA has been shown to be oxidized to the highly fluorescent 2,7-dichlorofluorescein in the presence of ROS. The neurons were washed twice with PBS, and then incubated with $10 \mathrm{mM}$ DCFH-DA for $30 \mathrm{~min}$ at $37^{\circ} \mathrm{C}$ in the dark. The cells were then harvested and suspended in PBS. The fluorescence intensity was measured by a fluorospectrophotometer (Hitachi, Tokyo, Japan) at excitation/emission maxima of 485/525 $\mathrm{nm}$ (20).

Measurement of $\left[\mathrm{Ca}^{2+}\right]_{i}$. After exposure to OGD/R, the cells were harvested, washed with PBS, centrifuged at 1,000 rpm for $5 \mathrm{~min}$, and then resuspended in PBS. Thereafter, the cell suspensions were incubated with $5 \mu \mathrm{M}$ Fluo-3 AM in the dark at $37^{\circ} \mathrm{C}$ for $40 \mathrm{~min}$. After centrifugation and one wash, the cells were resuspended in PBS. The fluorescence of $1 \times 10^{4}$ cells was analyzed by flow cytometry at $488 \mathrm{~nm}$ and the relative fluorescence intensity of Fluo-3 was used as an indicator of the quantity of $\left[\mathrm{Ca}^{2+}\right]_{\mathrm{i}}$.

Measurement of MMP. The cationic dye, JC-1 was used to evaluate the loss of MMP in hippocampal cultures subjected to OGD/R under a fluorescence microscope (Olympus, Tokyo, Japan). JC-1 is a convenient voltage sensitive probe used to monitor MMP (21). The cells containing J-aggregates have high $\Delta \psi_{\mathrm{m}}$, and show fluorescence. The cells with low $\Delta \psi_{\mathrm{m}}$ are those in which JC-1 maintains (or reacquires) its monomeric form, and show only green fluorescence (22). The relative proportions of red and green fluorescence were used to measure the ratio of mitochondrial depolarization.

Western blot analysis. The cell lysates were collected and protein concentrations were determined using the Pierce BCA Protein assay kit. Equal amounts of protein were separated by SDS-PAGE and transferred onto PVDF membranes. The membranes were blocked in 5\% nonfat milk in TBST buffer (5 mM Tris-HCl, pH 7.4, 136 mM NaCl, 0.1\% Tween-20) for $1 \mathrm{~h}$ at room temperature prior to hybridization with a primary antibody overnight at $4^{\circ} \mathrm{C}$, followed by three washes for
5 min with TBST. Following incubation with HRP-conjugated secondary antibodies for $1 \mathrm{~h}$ at room temperature and three washes with TBST, the resultant protein bands were visualized by ECL reagents according to the manufacturer's instructions. The absorbance values of the target proteins were obtained through Gel-Pro Analyzer version 4.0 software (Media Cybernetics, Silver Spring, MD, USA).

Statistical analysis. Data are expressed as the means \pm SD of results derived from three independent experiments performed in triplicate. Statistical analysis was performed using the Student's t-test and ANOVA. A P-value $<0.05$ was considered to indicate a statistically significant difference.

\section{Results}

SA pre-treatment ameliorates OGD/R-induced loss of viable hippocampal neuronal cells. In order to examine the protective effects of SA against cell cytotoxicity caused by OGD/R, MTT and LDH assays were performed to assess the viability of hippocampal neurons. Compared with the blank control group, viability was significantly decreased after subjecting the cells to $\mathrm{OGD} / \mathrm{R}$, and this effect was reversed by pre-treatment with SA at concentrations of $0.1,1,10$, and $20 \mu \mathrm{M}$, in a dose-dependent manner (Fig. 1A). In addition, LDH leakage was increased after $\mathrm{OGD} / \mathrm{R}$, and incubation with SA attenuated OGD/R-induced LDH leakage at concentrations of $0.1,1,10$, and $20 \mu \mathrm{M}$ in a dose-dependent manner (Fig. 1B). These results demonstrated that pre-treatment with SA attenuated the OGD/R-induced loss of viable hippocampal neuronal cells.

SA pre-treatment ameliorates $O G D / R$-induced oxidative stress in hippocampal neuronal cells. We next determined the effects of SA on OGD/R-induced oxidative stress in hippocampal neurons. OGD exposure markedly reduced the antioxidant SOD activity in the neuronal cultures, whereas SA $(0.1,1,10$ and $20 \mu \mathrm{M}$ ) pre-treatment resulted in a noticeable enhancement of SOD activity in a dose-dependent manner (Fig. 2A). Additionally, a 2.3-fold increase in intracellular ROS generation and a 3.2-fold elevation in MDA levels (a cellular lipid peroxidation product) were found after subjecting the hippocampal neuronal cells to OGD/R. Conversely, pre-treatment with SA $(0.1,1,10$, and $20 \mu \mathrm{M})$ decreased MDA content and ROS production in a dose-dependent manner (Fig. 2B and C). Taken together, these findings demonstrate that SA pre-treatment distinctly ameliorated OGD/R-induced oxidative stress in hippocampal neuronal cells.

SA pre-treatment prevents $O G D / R$-induced elevations in $\left[\mathrm{Ca}^{2+}\right]_{i}$ and MMP dissipation in hippocampal neuronal cells. Compared with the blank control group, $\left[\mathrm{Ca}^{2+}\right]_{\mathrm{i}}$ in the hippocampal neurons was significantly enhanced after exposure to OGD/R. However, nimodipine and SA $(0.1,1,10$, and $20 \mu \mathrm{M})$ pre-treatment decreased the enhanced $\left[\mathrm{Ca}^{2+}\right]_{\mathrm{i}}$ in a dose-dependent manner (Fig. 3A). Moreover, levels in the $20 \mu \mathrm{M}$ SA-treated group decreased to a similar level as those in the nimodipine-treated group.

JC-1 was used to assess the extent of mitochondrial depolarization in the hippocampal cultures exposed to OGD/R. After OGD/R, the red/green fluorescence intensity of JC-1 in 

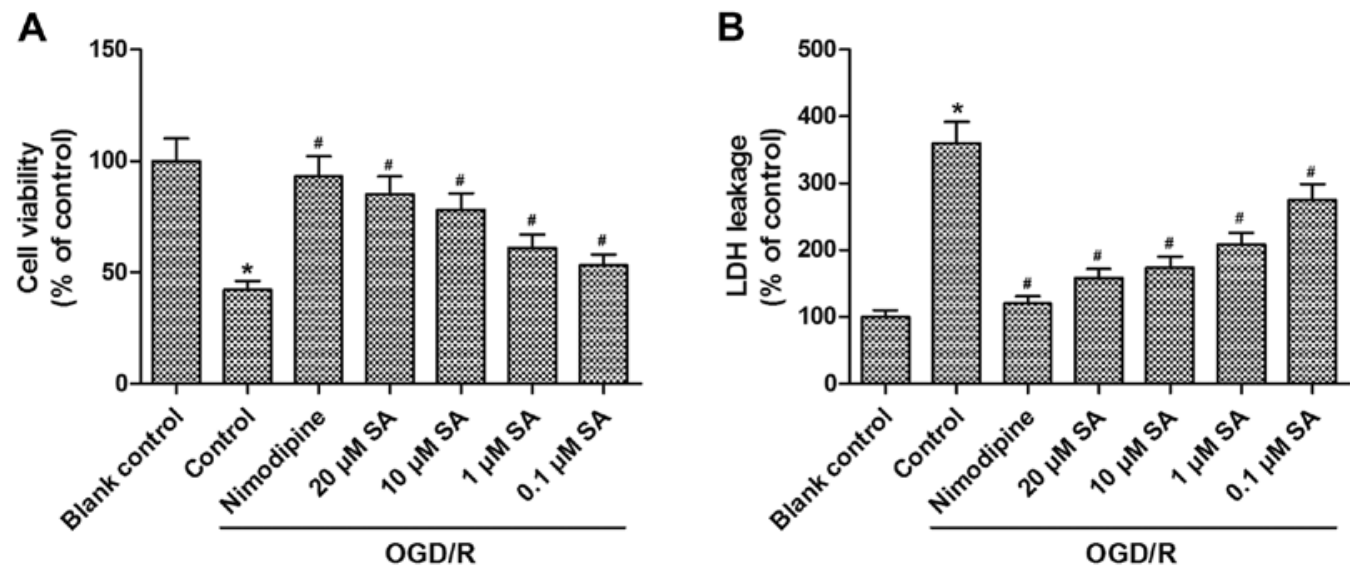

Figure 1. Effects of syringic acid (SA) on the viability of primary hippocampal neuronal cells subjected to oxygen-glucose deprivation (OGD) for $3 \mathrm{~h}$ followed by reperfusion for $24 \mathrm{~h}(\mathrm{OGD} / \mathrm{R})$. (A) Cell viability was determined using the MTT assay. (B) Lactate dehydrogenase (LDH) leakage was determined using a commercial LDH kit. ${ }^{*} \mathrm{P}<0.05$ vs. blank control group, ${ }^{\text {P }}<0.05$ vs. control group.

A
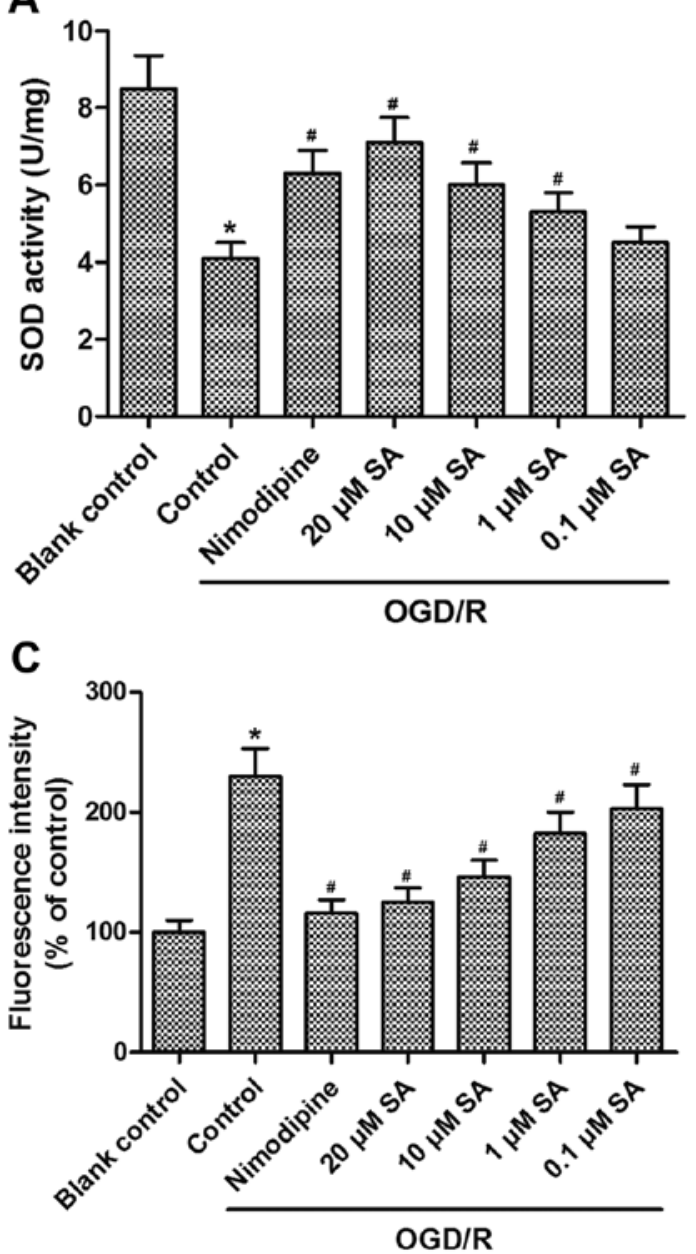

B

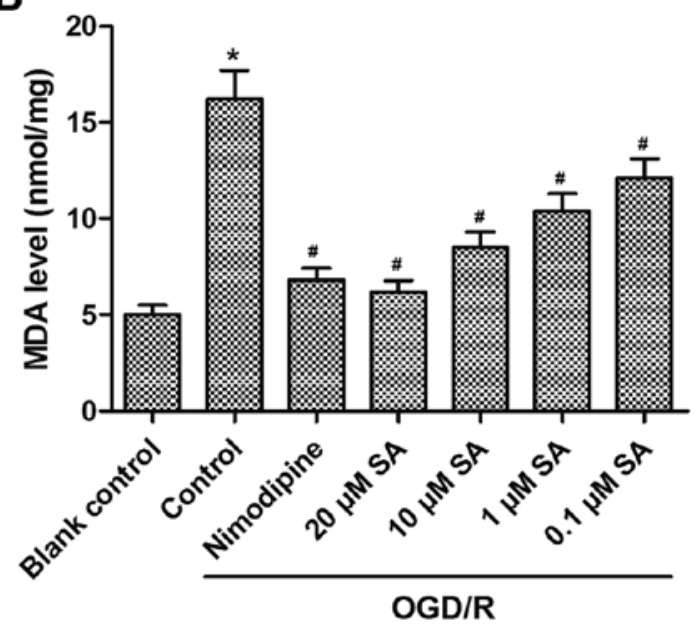

Figure 2. Effects of syringic acid (SA) on the levels of superoxide dismutase (SOD), malondialdehyde (MDA) and reactive oxygen species (ROS) in primary hippocampal neuronal cells subjected to oxygen-glucose deprivation (OGD) for $3 \mathrm{~h}$ followed by reperfusion for $24 \mathrm{~h}$ (OGD/R). Markers of oxidative status, (A) SOD, (B) MDA and (C) ROS, were examined using commercial kits. ${ }^{*} \mathrm{P}<0.05$ vs. blank control group, ${ }^{*} \mathrm{P}<0.05$ vs. control group.

the hippocampal neurons was reduced, suggesting a dissipation of the MMP whereas pre-treatment with SA $(0.1,1,10$, and $20 \mu \mathrm{M})$ significantly stabilized the MMP in a dose-dependent manner (Fig. 3B). Moreover, a greater stabilizing effect was achieved in the $20 \mu \mathrm{M} \mathrm{SA}$-treated group than in the other groups.
SA pre-treatment attenuates $O G D / R$-induced apoptosis of hippocampal neuronal cells. Western blot analysis revealed that exposure to OGD resulted in the apoptosis of hippocampal neurons, accompanied by a marked increase in the expression of Bax and caspase- 3 proteins, and a significant 


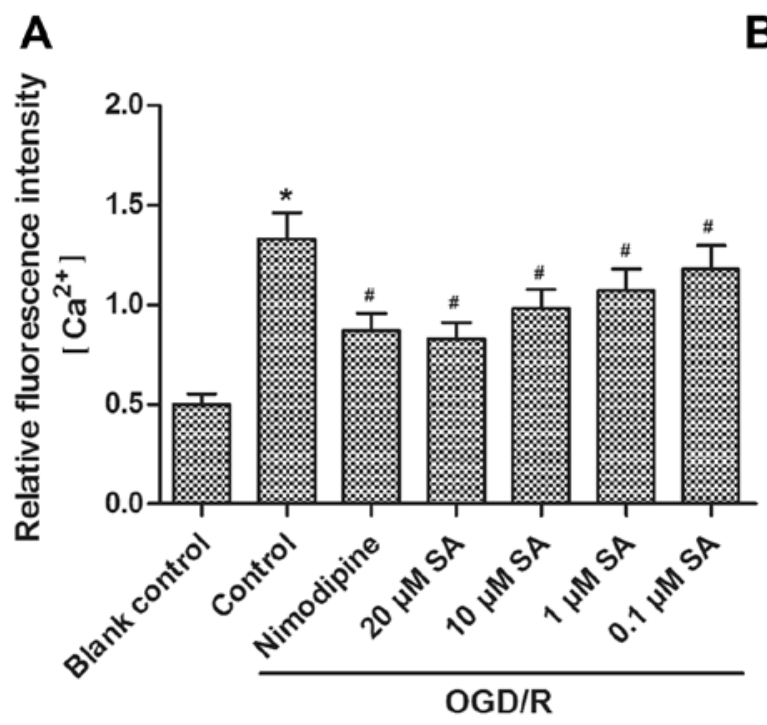

B
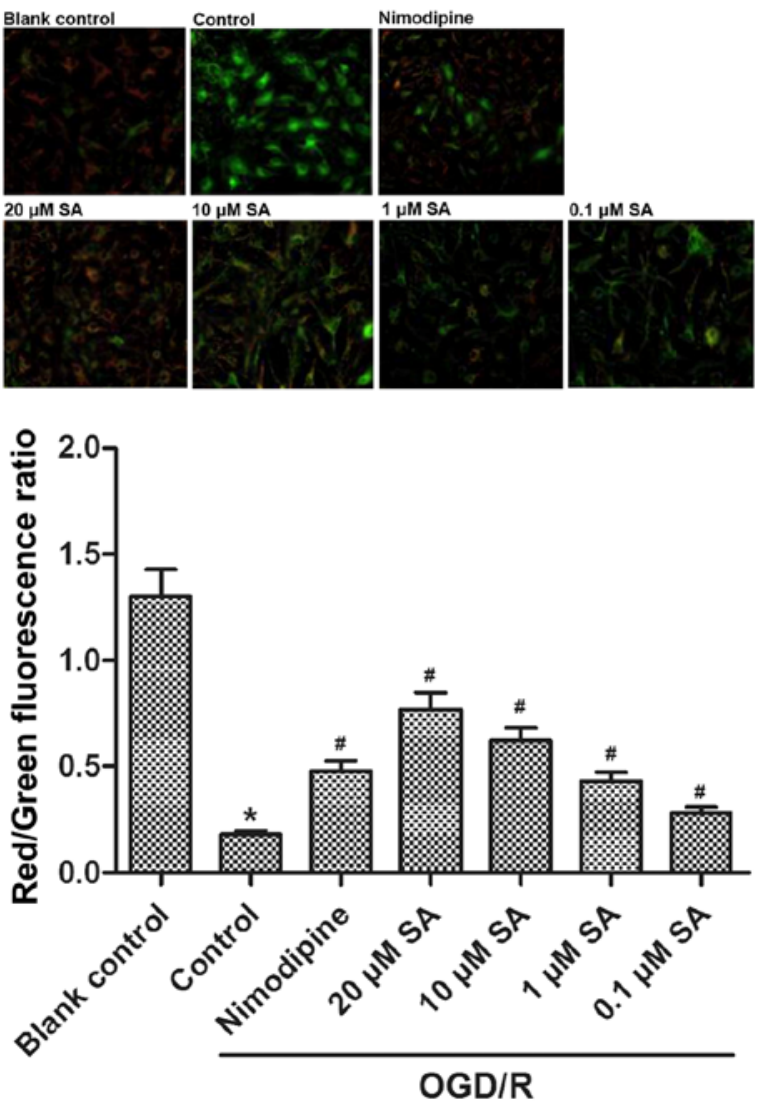

Figure 3. Effects of syringic acid (SA) on the concentration of intracellular-free calcium $\left[\mathrm{Ca}^{2+}\right]_{\mathrm{i}}$ and mitochondrial membrane potential $\left(\mathrm{MMP}\right.$ or $\left.\Delta \psi_{\mathrm{m}}\right)$ in primary hippocampal neuronal cells subjected to oxygen-glucose deprivation (OGD) for $3 \mathrm{~h}$ followed by reperfusion for $24 \mathrm{~h}(\mathrm{OGD} / \mathrm{R})$. (A) [Ca $\left.{ }^{2+}\right]_{i}$ was measured by flow cytometric analysis. The relative fluorescence intensity of Fluo-3 was used as an indicator of $\left[\mathrm{Ca}^{2+}\right]_{\mathrm{i}}$. (B) The intracellular red and green fluorescence of JC-1 was determined under a fluorescent inverted microscope. Red fluorescence indicated polarized mitochondrial membranes, whereas green fluorescence indicated depolarized mitochondrial membranes. Magnification, x200. A representative histogram of MMP changes is shown. " $\mathrm{P}<0.05$ vs. blank control group, ${ }^{\#} \mathrm{P}<0.05$ vs. control group.

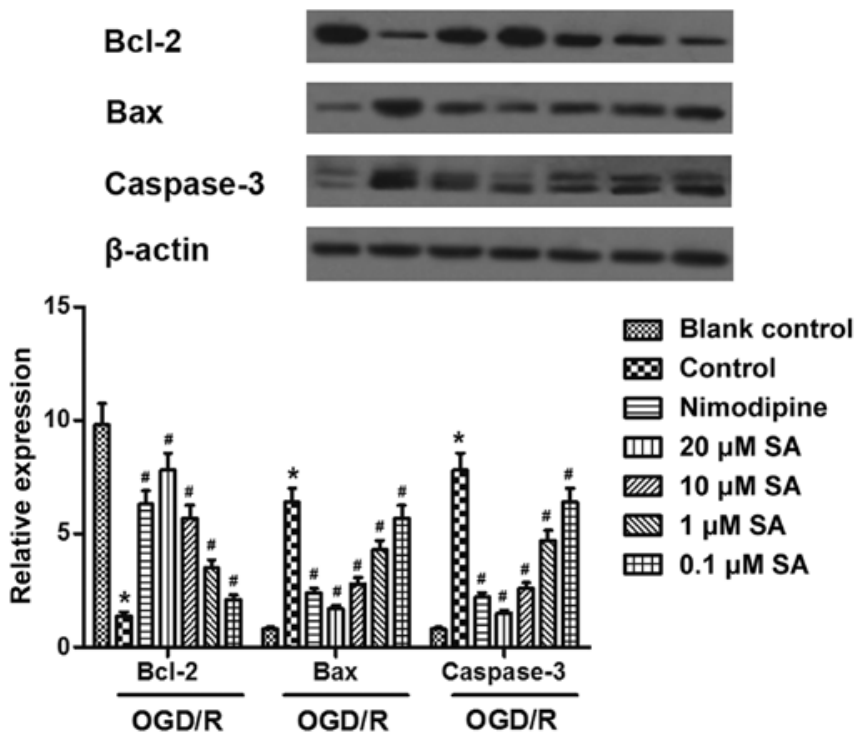

Figure 4. Effects of syringic acid (SA) on the expression of apoptosis-related proteins in primary hippocampal neuronal cells subjected to oxygen-glucose deprivation (OGD) for $3 \mathrm{~h}$ followed by reperfusion for $24 \mathrm{~h}$ (OGD/R). The expression of Bcl-2, Bax, caspase- 3 and $\beta$-actin proteins was evaluated by western blot analysis and representative blots are shown. Quantification analysis was performed using Gel-Pro Analyzer version 4.0 software. ${ }^{*} \mathrm{P}<0.05$ vs. blank control group, ${ }^{\#} \mathrm{P}<0.05$ vs. control group. reduction in Bcl-2 protein. However, in the SA $(0.1,1,10$, and $20 \mu \mathrm{M}$ )-pre-treated groups, the expression of Bax and caspase-3 was downregulated, and the expression of Bcl-2 was upregulated in a dose-dependent manner (Fig. 4). Thus, these data suggested that SA pre-treatment attenuated the OGD/R-induced apoptosis of hippocampal neuronal cells.

SA pre-treatment inhibits $O G D / R$-induced activation of $J N K$ and p38 signaling pathways in hippocampal neuronal cells. Furthermore, we examined whether the JNK and p38 pathways are associated with the neuroprotective effects of SA against OGD/R-induced injury in neuronal cells. Western blot analysis revealed that the levels of phosphorylated (p-)JNK and p-p38 expression were enhanced by exposure to OGD/R, and inhibited by pre-treatment with SA in a dose-dependent manner (Fig. 5). The results indicated that SA pre-treatment inhibited the OGD/R-induced activation of the JNK and p38 signaling pathways in hippocampal neuronal cells.

\section{Discussion}

Cerebral ischemia is one of the most common causes of death worldwide after cardiovascular diseases and cancer, with a higher incidence in aged individuals (23). The therapeutic aim 


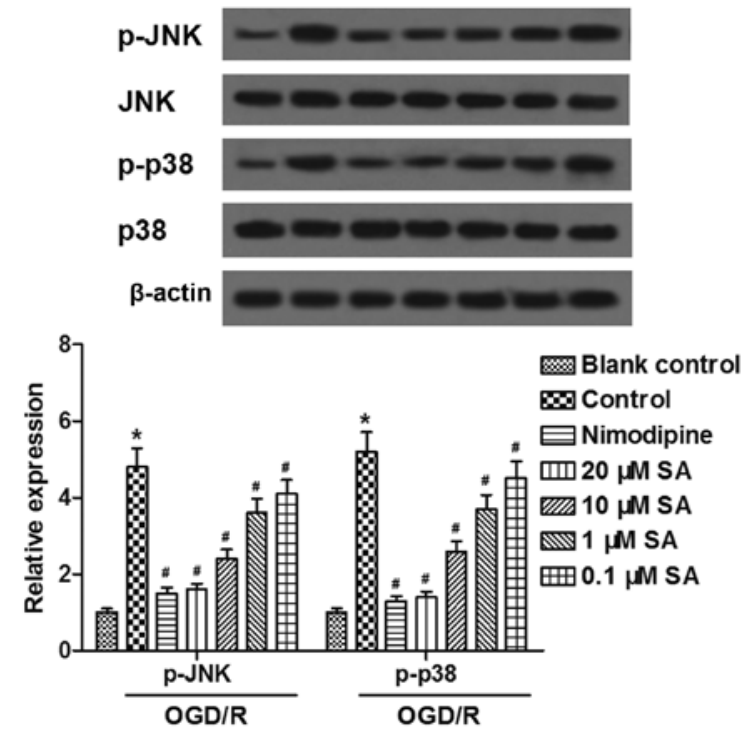

Figure 5. Effects of syringic acid (SA) on the activation of JNK and p38 signaling pathways in primary hippocampal neuronal cells subjected to oxygen-glucose deprivation (OGD) treatment for $3 \mathrm{~h}$ followed by reperfusion for $24 \mathrm{~h}(\mathrm{OGD} / \mathrm{R})$. The expression of phosphorylated (p-)JNK, JNK, p-p38 and p38 proteins was detected by western blot analysis and representative blots are shown. Quantification analysis was performed using Gel-Pro Analyzer version 4.0 software. ${ }^{*} \mathrm{P}<0.05$ vs. blank control group, ${ }^{\#} \mathrm{P}<0.05$ vs. control group.

in treating cerebral ischemia is to reduce the extent of brain injury and thus, minimize neurological impairment. In order to develop effective strategies for the prevention, treatment and prognosis of the disease, it is necessary to identify safe and effective drugs or natural substances and furthermore, to have a sufficient understanding of the pathological mechanisms involved in cerebral ischemia/reperfusion injury. In the present study, we examined the protective effects of SA in primary cultures of hippocampal neurons injured by OGD/R.

Studies have shown that antioxidants reduce ischemic injury in the brain. Indeed, the efficacy of plant extracts, including gallic acid, (S)-ZJM-289, aloperine and SA, in the prevention of stroke and cerebral ischemia has been evaluated in some cell and animal models of ischemic insults (12,24-26). In particular, $\mathrm{SA}$ is an active compound isolated from Isatis indigotica or Radix isatidis, which has been demonstrated to exert strong antioxidant and hepatoprotective effects $(11,27)$. On the other hand, it is well known that ROS have been associated with high levels of MDA and suggested to cause lipid peroxidation thereby initiating the cascade of cell membrane damage. The synthesis of SOD is the principal factor involved in controlling ROS levels. Previous findings have demonstrated that SA is a strong inhibitor of low-density lipoprotein oxidation, contributing to the scavenging of free radicals, reducing the production of MDA, and thus, slowing the development of atherosclerosis (28). SA reduces the levels of oxidative stress markers and exerts antioxidant effects, augmenting the antioxidant capacity in L-arginine-induced acute pancreatitis in rats (11). In the present study, we found that pre-treatment with SA ameliorated OGD/R-induced oxidative stress in hippocampal neuronal cells in a dose-dependent manner.

Caspase-3 is a major cell death effector protease in the adult and neonatal nervous system (29), which is crucial among multiple distinct caspases during neuronal develop- ment and under pathological conditions including cerebral ischemia (30). As a neuroprotective agent, SA reduced oxidative stress and neuronal degeneration, with increased SOD generation and decreased levels of MDA and caspase-3, in rats with spinal cord ischemia/reperfusion (12) or in rats with cerebral ischemia (9). Moreover, phenolic acids, including SA, exerted distinct inhibitory effects on the viability, cytotoxicity and apoptosis of Neuro-2A cells induced by methylglyoxal (31). Similarly, our data revealed that pre-treatment with SA dose-dependently attenuated the OGD/R-induced loss of viability and apoptosis of hippocampal neuronal cells, suggesting that SA may exert cytoprotective effects against OGD/R-induced neuronal injury.

To further explore the mechanisms through which SA protects against $\mathrm{OGD} / \mathrm{R}$ injury, we performed additional experiments measuring $\left[\mathrm{Ca}^{2+}\right]_{\mathrm{i}}$ and MMP. Calcium is an important second messenger involved in neurotransmitter release and signal transduction. Numerous studies have definitely indicated that alterations in intracellular $\mathrm{Ca}^{2+}$ homeostasis play a central role in initiating the apoptotic response (32). In addition, it has been suggested that mitochondrial membrane depolarization is a determining factor in the final step to apoptosis (7). Previously, both elevations of $\left[\mathrm{Ca}^{2+}\right]_{i}$ and MMP loss lead to destabilization of the neuronal cell structure and caused cell damage, eventually leading to cell death $(7,26)$. Polyphenols from the plant Zanthoxylum heitzii, including SA as a major component, exerted an inhibitory effect on HL-60 cells through ROS generation, MMP loss and cell cycle destabilization (33). Similarly, in our experiments, the results demonstrated that SA significantly reduced $\left[\mathrm{Ca}^{2+}\right]_{\mathrm{i}}$ elevation, whereas it increased the MMP after OGD/R exposure in hippocampal neuronal cells, inferring that the antioxidant effects of SA in cultured hippocampal neurons injured by OGD/R may be associated with the inhibition of $\left[\mathrm{Ca}^{2+}\right]_{i}$ overload and the stabilization of the MMP.

As suggested by previous findings, OGD/R induced the activation of the apoptotic signaling pathways of JNK and p38, and increased the activity of caspase- 3 in primary cultures of rat cortical neuronal cells (34). In addition, the activation of JNK and p38 signaling pathways participated in the methylglyoxal-induced apoptosis of Neuro-2A cells (31), which was inhibited by pre-treatment with SA. Our data demonstrated that SA inhibited the OGD/R-induced activation of JNK and p38 signaling pathways in hippocampal neuronal cells.

In conclusion, the data revealed that SA exerted strong neuroprotective effects against OGD/R-induced neuronal injury in vitro. Our results demonstrated that pre-treatment with SA may inhibit the OGD/R-induced loss of viability, oxidative stress, $\left[\mathrm{Ca}^{2+}\right]_{\mathrm{i}}$ overload, MMP dissipation and apoptosis of cultured hippocampal neuronal cells through the JNK and $\mathrm{p} 38$ signaling pathways.

\section{References}

1. Donnan GA, Fisher M, Macleod M and Davis SM: Stroke. Lancet 371: 1612-1623, 2008.

2. Chen H, Yoshioka H, Kim GS, Jung JE, Okami N, Sakata H, Maier CM, Narasimhan P, Goeders CE and Chan PH: Oxidative stress in ischemic brain damage: mechanisms of cell death and potential molecular targets for neuroprotection. Antioxid Redox Signal 14: 1505-1517, 2011.

3. Schmidt-Kastner R and Freund TF: Selective vulnerability of the hippocampus in brain ischemia. Neuroscience 40: 599-636, 1991. 
4. Christophe M and Nicolas S: Mitochondria: a target for neuroprotective interventions in cerebral ischemia-reperfusion. Curr Pharm Des 12: 739-757, 2006.

5. Turrens JF: Mitochondrial formation of reactive oxygen species. J Physiol 552: 335-344, 2003.

6. Radermacher KA, Wingler K, Langhauser F, Altenhöfer S, Kleikers P, Hermans JJ, Hrabě de Angelis M, Kleinschnitz C and Schmidt HH: Neuroprotection after stroke by targeting NOX4 as a source of oxidative stress. Antioxid Redox Signal 18: 1418-1427, 2013.

7. Rui C, Yuxiang L, Yinju H, Qingluan Z, Yang W, Qipeng Z, Hao W, Lin M, Juan L, Chengjun Z, et al: Protective effects of Lycium barbarum polysaccharide on neonatal rat primary cultured hippocampal neurons injured by oxygen-glucose deprivation and reperfusion. J Mol Histol 43: 535-542, 2012.

8. Scalbert A and Williamson G: Dietary intake and bioavailability of polyphenols. J Nutr 130 (8S Suppl): 2073S-2085S, 2000.

9. Güven M, Aras AB, Topaloğlu N, Ozkan A, Sen HM, Kalkan Y, Okuyucu A, Akbal A, Gökmen F and Coşar M: The protective effect of syringic acid on ischemia injury in rat brain. Turk J Med Sci 45: 233-240, 2015.

10. Kumar S, Prahalathan P and Raja B: Syringic acid ameliorates (L)-NAME-induced hypertension by reducing oxidative stress. Naunyn Schmiedebergs Arch Pharmacol 385: 1175-1184, 2012.

11. Cikman O, Soylemez O, Ozkan OF, Kiraz HA, Sayar I, Ademoglu S, Taysi S and Karaayvaz M: Antioxidant activity of syringic acid prevents oxidative stress in L-arginine-induced acute pancreatitis: an experimental study on rats. Int Surg 100: 891-896, 2015

12. Tokmak M, Yuksel Y, Sehitoglu MH, Guven M, Akman T, Aras AB, Cosar M and Abbed KM: The neuroprotective effect of syringic acid on spinal cord ischemia/reperfusion injury in rats Inflammation 38: 1969-1978, 2015.

13. Kazda S and Towart R: Nimodipine: a new calcium antagonistic drug with a preferential cerebrovascular action. Acta Neurochir (Wien) 63: 259-265, 1982.

14. Scriabine A and van den Kerckhoff W: Pharmacology of nimodipine. A review. Ann N Y Acad Sci 522: 698-706, 1988.

15. Wadworth AN and McTavish D: Nimodipine. A review of its pharmacological properties, and therapeutic efficacy in cerebral disorders. Drugs Aging 2: 262-286, 1992.

16. Krohn AJ, Preis E and Prehn JH: Staurosporine-induced apoptosis of cultured rat hippocampal neurons involves caspase-1-like proteases as upstream initiators and increased production of superoxide as a main downstream effector. J Neurosci 18: 8186-8197, 1998

17. McCord JM and Fridovich I: Superoxide dismutase. An enzymic function for erythrocuprein (hemocuprein). J Biol Chem 244: 6049-6055, 1969.

18. Gwag BJ, Lobner D, Koh JY, Wie MB and Choi DW: Blockade of glutamate receptors unmasks neuronal apoptosis after oxygen-glucose deprivation in vitro. Neuroscience 68: 615-619, 1995.

19. Ohkawa H, Ohishi N and Yagi K: Assay for lipid peroxides in animal tissues by thiobarbituric acid reaction. Anal Biochem 95 351-358, 1979 .

20. Wu W, Xia Q, Luo RJ, Lin ZQ and Xue P: In vitro study of the antagonistic effect of low-dose liquiritigenin on gemcitabine-induced capillary leak syndrome in pancreatic adenocarcinoma via inhibiting ROS-mediated signalling pathways. Asian Pac J Cancer Prev 16: 4369-4376, 2015.
21. Reers M, Smith TW and Chen LB: J-aggregate formation of a carbocyanine as a quantitative fluorescent indicator of membrane potential. Biochemistry 30: 4480-4486, 1991.

22. Zhu XJ, Shi Y, Peng J, Guo CS, Shan NN, Qin P, Ji XB and Hou M: The effects of BAFF and BAFF-R-Fc fusion protein in immune thrombocytopenia. Blood 114: 5362-5367, 2009.

23. Margaill I, Plotkine M and Lerouet D: Antioxidant strategies in the treatment of stroke. Free Radic Biol Med 39: 429-443, 2005.

24. Sun J, Li YZ, Ding YH, Wang J, Geng J, Yang H, Ren J, Tang JY and Gao J: Neuroprotective effects of gallic acid against hypoxia/reoxygenation-induced mitochondrial dysfunctions in vitro and cerebral ischemia/reperfusion injury in vivo. Brain Res 1589: 126-139, 2014.

25. Zhang C, Zhang Z, Zhao Q, Wang X, Ji H and Zhang Y: (S)-ZJM-289 preconditioning induces a late phase protection against nervous injury induced by transient cerebral ischemia and oxygen-glucose deprivation. Neurotox Res 26: 16-31, 2014.

26. Ma NT, Zhou R, Chang RY, Hao YJ, Ma L, Jin SJ, Du J, Zheng J, Zhao CJ, Niu Y, et al: Protective effects of aloperine on neonatal rat primary cultured hippocampal neurons injured by oxygen-glucose deprivation and reperfusion. J Nat Med 69: 575-583, 2015.

27. Itoh A, Isoda K, Kondoh M, Kawase M, Watari A, Kobayashi M, Tamesada M and Yagi K: Hepatoprotective effect of syringic acid and vanillic acid on CCl4-induced liver injury. Biol Pharm Bull 33: 983-987, 2010.

28. Morton LW, Croft KD, Puddey IB and Byrne L: Phenolic acids protect low density lipoproteins from peroxynitrite-mediated modification in vitro. Redox Rep 5: 124-125, 2000.

29. Le DA, Wu Y, Huang Z, Matsushita K, Plesnila N, Augustinack JC, Hyman BT, Yuan J, Kuida K, Flavell RA and Moskowitz MA: Caspase activation and neuroprotection in caspase-3-deficient mice after in vivo cerebral ischemia and in vitro oxygen glucose deprivation. Proc Natl Acad Sci USA 99: 15188-15193, 2002.

30. Nicholson DW: Caspase structure, proteolytic substrates, and function during apoptotic cell death. Cell Death Differ 6: 1028-1042, 1999.

31. Huang SM, Chuang HC, Wu CH and Yen GC: Cytoprotective effects of phenolic acids on methylglyoxal-induced apoptosis in Neuro-2A cells. Mol Nutr Food Res 52: 940-949, 2008.

32. Orrenius S, Zhivotovsky B and Nicotera P: Regulation of cell death: the calcium-apoptosis link. Nat Rev Mol Cell Biol 4: 552-565, 2003

33. Pieme CA, Santosh GK, Tekwu EM, Askun T, Aydeniz H, Ngogang JY, Bhushan S and Saxena AK: Fruits and barks extracts of Zanthoxylum heitzii a spice from Cameroon induce mitochondrial dependent apoptosis and Go/G1 phase arrest in human leukemia HL-60 cells. Biol Res 47: 54, 2014.

34. Wang CP, Li JL, Zhang LZ, Zhang XC, Yu S, Liang XM, Ding F and Wang ZW: Isoquercetin protects cortical neurons from oxygen-glucose deprivation-reperfusion induced injury via suppression of TLR4-NF- $\mathrm{KB}$ signal pathway. Neurochem Int 63: 741-749, 2013. 